\title{
AVALIAÇÃO DA ANASTOMOSE ESPLENO-RENAL DISTAL, COM E SEM LIGADURA DA ARTÉRIA ESPLÊNICA, PARA TRATAMENTO DA HIPERTENSÃO PORTAL ESQUISTOSSOMÓTICA
}

\author{
EVALUATION OF DISTAL SPLENORENAL SHUNT, WITH AND WITHOUT \\ SPLENIC ARTERY LIGATION, FOR THE TREATMENT OF SCHISTOSOMAL \\ PORTAL HYPERTENSION
}

\author{
Valdinaldo Aragão de Melo, TCBC-SE ${ }^{1}$ \\ Gustavo Barreto de Melo ${ }^{2}$ \\ Reginaldo Ceneviva, TCBC-SP ${ }^{3}$
}

\begin{abstract}
RESUMO: Objetivo: Avaliar os resultados da derivação espleno-renal distal (DERD) com ligadura da artéria esplênica (LAE) em pacientes com hipertensão portal esquistossomótica e história de sangramento por varizes esôfago-gástricas. Método: Estudo prospectivo de trinta pacientes que foram divididos em dois grupos: 15 foram submetidos à DERD (Grupo I) e 15 foram submetidos à DERD associada à LAE (Grupo II). Os pacientes foram acompanhados por 24 meses, e estudados quanto à recorrência de hemorragia digestiva por ruptura de varizes, controle endoscópico das varizes e permeabilidade da anastomose através de ultrasonografia e angiografia. Resultados: Um paciente do Grupo I $(6,67 \%)$ apresentou trombose da anastomose e recidiva hemorrágica em decorrência de varizes. No Grupo II, nenhum paciente, em dois anos de observação, desenvolveu trombose da anastomose e hemorragia digestiva. Não houve diferença estatisticamente significativa entre os grupos. Quanto à análise endoscópica após seis meses, houve redução do tamanho ou desaparecimento das varizes em $80 \%$ dos pacientes do Grupo I e em 93\% daqueles do Grupo II. Conclusões: A derivação espleno-renal distal com ligadura da artéria esplênica não está associada a uma maior incidência de trombose da anastomose, de recidiva hemorrágica e nem modificou o tamanho das varizes esôfagogástricas em comparação com a derivação espleno-renal distal isolada.
\end{abstract}

Descritores: Derivação espleno-renal cirúrgica; Hipertensão portal; Esquistossomose; Trombose; Recidiva; Hemorragia; Artéria esplênica.

\section{INTRODUÇÃO}

A esquistossomose é um problema de saúde pública relevante em todo o mundo, afetando mais de 200 milhões de pessoas na África, Ásia, América do Sul e Caribe ${ }^{1}$. No Brasil, estima-se que haja entre seis e sete milhões de portadores desta patologia². Os pacientes que apresentam a forma hepatoesplênica da doença desenvolvem fibrose periportal de Symmers com alteração mínima da função hepática ${ }^{3}$. A princi- pal complicação é a hemorragia digestiva por ruptura de varizes, que pode levar à morte.

O tratamento clínico e endoscópico consiste de escleroterapia, ligadura elástica e uso de betabloqueadores ${ }^{3}$. Um grande número de procedimentos cirúrgicos (derivativos e não-derivativos) foi desenvolvido. O objetivo da derivação é evitar a hemorragia varicosa ao desviar o fluxo da veia porta para a circulação sistêmica. Conseqüentemente, há diminuição da pressão portal ${ }^{4}$. A anastomose porto-

1. Professor Adjunto do Departamento de Medicina da Universidade Federal de Sergipe (UFS).

2. Acadêmico de Medicina da Universidade Federal de Sergipe (UFS).

3. Professor Titular do Departamento de Cirurgia e Anatomia da Faculdade de Medicina de Ribeirão Preto da USP.

Recebido em 05/06/2003

Aceito para publicação em 14/10/2003

Trabalho realizado na Faculdade de Medicina de Ribeirão Preto da Universidade de São Paulo e na Faculdade de Medicina da Universidade Federal de Sergipe. 
cava é um procedimento eficiente para reduzir a hemorragia digestiva alta, mas provoca alta incidência de encefalopatia porto-sistêmica ${ }^{3}$. Na tentativa de manter os bons resultados sem os efeitos colaterais indesejados, a derivação espleno-renal distal (DERD) foi desenvolvida ${ }^{5}$. Baixos índices de recidiva hemorrágica e de encefalopatia, assim como maior sobrevida, são obtidos com esta técnica ${ }^{6}$.

Em alguns pacientes, associada à derivação espleno-renal distal, foi realizada também a ligadura da artéria esplênica (LAE) a fim de promover a melhora do hiperesplenismo. Entretanto, não há trabalhos que definam a indicação e as complicações desta técnica.Objetivou-se, então, estudar a derivação espleno-renal distal isolada e com a ligadura da artéria esplênica, em pacientes com hipertensão portal esquistossomótica, comparando a permeabilidade da anastomose, recidiva hemorrágica e controle endoscópico das varizes após dois anos de acompanhamento pós-operatório.

\section{MÉTODO}

Trinta pacientes com hipertensão portal esquistossomótica e história de hemorragia digestiva alta por ruptura de varizes, aleatoriamente escolhidos para DERD com ou sem LAE, foram avaliados por dois anos após a cirurgia. O diagnóstico de esquistossomose foi baseado em dados epidemiológicos, clínicos e parasitológicos e foi confirmado por análise histopatológica de fragmento de fígado biopsiado no pré-operatório ou durante a cirurgia. Pacientes com outras doenças hepáticas e sistêmicas que pudessem alterar os resultados foram excluídos do estudo.

O Grupo I consistiu de 15 pacientes que se submeteram somente à DERD e o Grupo II, de 15 pacientes que foram operados de DERD e LAE. A média de idade foi de 42,46 \pm 13,09 anos no Grupo I e $35,86 \pm 12,56$ no Grupo II.

A DERD foi realizada por uma incisão mediana e dissecção da veia esplênica até a sua junção com a veia mesentérica superior ${ }^{5}$. Foi feita uma abertura no peritônio posterior para abordagem da veia renal esquerda e realização de anastomose términolateral com o coto da veia esplênica. A ligadura das veias gástrica esquerda e gastroepiplóica direita foi realizada, assim como biópsia hepática para confirmação histopatológica. Nos pacientes do Grupo II, a artéria esplênica foi ligada.

Todos os pacientes foram observados durante dois anos após a cirurgia. Os parâmetros analisados foram: recidiva hemorrágica, controle endoscópico das varizes esôfago-gástricas e trombose da anastomose. A recorrência de sangramento varicoso foi analisada clinicamente durante todo o período de observação. A endoscopia foi realizada no período pré-operatório e aos seis meses do pós-operatório. As varizes foram classificadas, de acordo com o calibre, em grandes (maior que $6 \mathrm{~mm}$ ), médias (entre 4 e $6 \mathrm{~mm}$ ) e pequenas (até $3 \mathrm{~mm}$ de diâmetro). A comparação entre os períodos analisados permitiu obter as seguintes variáveis sobre o calibre das varizes: aumento, redução, sem alteração e desaparecimento. O controle da perviedade da anastomose espleno-renal distal foi feito aos seis meses de pós-operatório através de ultra-sonografia e angiografia.

Para a análise estatística dos dados, foi utilizado o programa SPSS e realizados os testes de Wilcoxon para comparação do pré-operatório com pós-operatório e de Mann-Whitney para comparação dos dois grupos. O nível de significância considerado foi 0,05 .

\section{RESULTADOS}

Como pode ser evidenciado na Tabela 1, a endoscopia realizada após seis meses de pós-operatório, mostrou não ter havido redução no tamanho das varizes em 13,33\% dos pacientes do Grupo I e em 6,67\% daqueles do Grupo II; estavam menores em 80 e $93 \%$ dos pacientes, dos Grupos I e II, respectivamente; desapareceram em $13,33 \%$ dos pacientes do Grupo II; um paciente do Grupo I teve aumento no tamanho das varizes.

Tabela 1 - Avaliação endoscópica do calibre das varizes esofagianas após dois anos de pós-operatório.

\begin{tabular}{llccc}
\hline & Sem alteração & Redução & Desaparecimento & Aumento \\
\hline Grupo I & $2(13,33 \%)$ & $12(80 \%)$ & 0 & $1(6,66 \%)$ \\
Grupo II & $1(6,66 \%)$ & $12(80 \%)$ & $2(13,33 \%)$ & 0 \\
\hline
\end{tabular}


O paciente que desenvolveu aumento no diâmetro das varizes após a cirurgia apresentou recidiva hemorrágica $(6,67 \%)$ e trombose da anastomose. Entretanto, não houve diferença estatisticamente significante entre os resultados dos dois grupos analisados. A perviedade da anastomose dos pacientes estudados foi demonstrada através da ultra-sonografia e da angiografia (Figura 1).

\section{DISCUSSÃO}

A derivação espleno-renal distal pode ser considerada segura, duradoura e eficaz no controle da hemorragia digestiva por ruptura de varizes esôfago-gástricas em pacientes com hipertensão portal e boa função hepática ${ }^{7,8}$. Sua associação com ligadura da arté- dimento teve sua indicação proscrita devido aos altos índices de encefalopatia hepática. A associação da LAE à DERD, apesar de não ter apresentado significância estatística, aponta para uma tendência maior de redução no tamanho das varizes esôfago-gástricas.

Neste estudo, a incidência global de trombose e de recidiva hemorrágica foi $3,33 \%$. De maneira geral, as taxas de sangramento são menores que $10 \%$. O maior risco de sua ocorrência é no primeiro mês após a cirurgia, provavelmente relacionado à trombose da anastomose. Esta pode ser por erro técnico e requer, em alguns casos, reoperação. A associação entre as duas manifestações foi constatada em nosso trabalho. $\mathrm{O}$ paciente que a desenvolveu foi submetido a desconexão ázigo-portal e esplenectomia e se recuperou sem complicações.

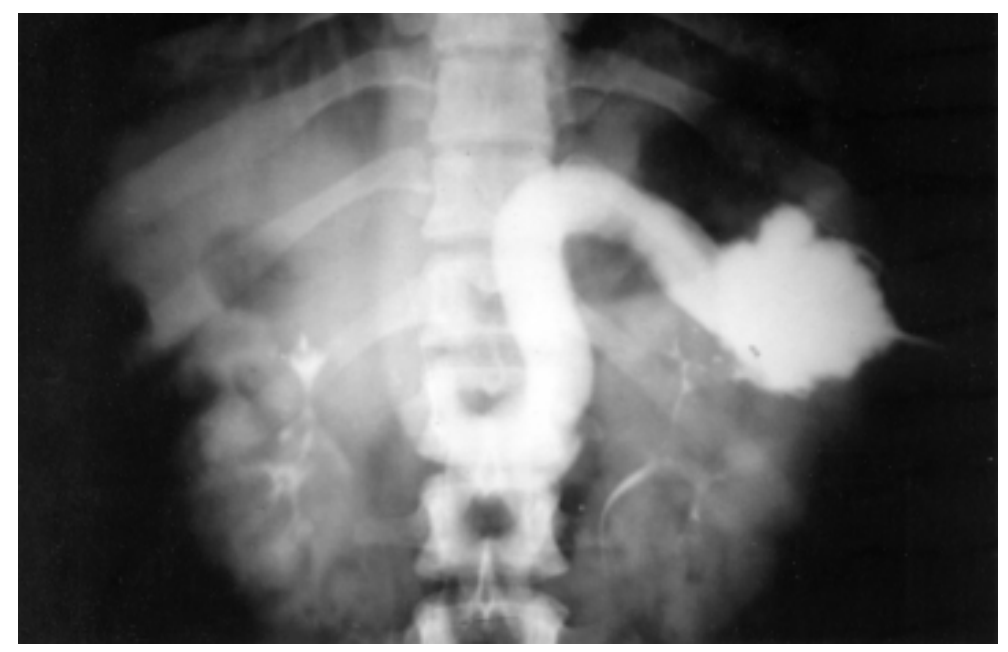

Figura 1 - Angiografia demonstrando a perviedade da anastomose.

ria esplênica tem sido realizada no Brasil em portadores de esquistossomose hepatoesplênica. As indicações para este procedimento são, principalmente, reduzir o tamanho do baço, melhorar o hiperesplenismo e a hipertensão pulmonar ${ }^{9,10}$.

Nos pacientes com hipertensão portal, varizes de grossos calibres são um grande fator de risco para hemorragia digestiva alta. Após um primeiro episódio, a chance de recidiva está entre 50 e $80 \%$. Além disso, observa-se que o índice de mortalidade do paciente com sangramento é de $30 \%$ a $50 \% \%^{8,11}$.

Neste trabalho, a DERD com ou sem LAE causou redução significativa no tamanho das varizes seis meses após a cirurgia. Em alguns trabalhos ${ }^{3,6,8}$, pode-se constatar que a DERD isolada é bastante eficiente nesta diminuição, mesmo sendo um pouco inferior que a derivação espleno-renal proximal ${ }^{3,8}$. Este último proce-
A taxa de perviedade da anastomose aumenta com o passar do tempo e com a experiência da equipe cirúrgica. Na série de Maksoud et al ${ }^{12}$, foi de $71 \%$ nos primeiros casos. Na fase seguinte, o índice passou para 95\%. Em alguns trabalhos encontrados na literatura, a incidência de trombose da anastomose varia de 0 a 14,3\% \%,13.

A ligadura da artéria esplênica associada à DERD não causou aumento na incidência de trombose nem de ressangramento. Jin et al $^{14}$, por outro lado, demonstraram uma elevada incidência de trombose da anastomose em pacientes submetidos à ligadura da artéria esplênica, com elevação da pressão das varizes, resultando em ressangramento.

Pode-se concluir que a ligadura da artéria esplênica associada à derivação espleno-renal distal não aumentou a incidência de trombose da anastomose nem a taxa de recidiva hemorrágica em nosso estudo. 


\begin{abstract}
Background: A prospective study was developed throughout two years of observation to assess the results of distal splenorenal shunt (DSRS) associated with splenic artery ligation (SAL) in patients with schistosomal portal hypertension and history of gastroesophageal varices bleeding. Methods: Thirty patients were divided into two groups: fifteen were submited to DSRS (Group I) and the other 15 were submited to DSRS associated with SAL (Group II). They were observed through 24 months of follow-up. In this period, it was assessed: recurrence of gastrointestinal bleeding, variceal endoscopic control and anastomosis patency at ultrasound and angiography. Results: One patient from Group I (6.67\%) developed thrombosis of the shunt and hemorrhage recurrence due to varices. In Group II, none of the patients, throughout 2 years of observation, disclosed anastomosis thrombosis and gastrointestinal bleeding. There was no statistically significant difference between the groups. Concerning with the endoscopic analysis six months after surgery, there was decrease in the size or disappearance of varices in $80 \%$ of patients from Group I and in $93 \%$ of those from Group II. Conclusions: Distal splenorenal shunt with splenic artery ligation is not associated with a higher incidence of thrombosis of the anastomosis, hemorrhage recurrence and did not change gastroesophageal variceal size in comparison to distal splenorenal shunt isolated.
\end{abstract}

Key Words: Splenorenal shunt, surgical; Hypertension, portal; Schistosomiasis; Thrombosis; Hemorrhage; Recurrence; Splenic artery.

\section{REFERÊNCIAS}

1. Warren KS - The relevance of schistosomiasis. N Engl J Med, 1998, 303: 203-206.

2. Katz N, Peixoto SV - Análise crítica da estimativa do número de portadores de esquistossomose mansoni no Brasil. Rev Soc Bras Med Trop, 2000, 33(3): 303-308.

3. Strauss E, Sakai P, Gayotto LCC, et al. - Size of gastroesophageal varices: its behavior after the surgical treatment of portal hypertension. Rev Hosp Clin Fac Med Univ São Paulo, 1999, 54(6):193-198.

4. Tajiri T, Onda M, Yoshida H, et al. - Comparison of the long-term results of distal splenorenal shunt and esophageal transection for the treatment of esophageal varices. Hepatogastroenterology, 2000, 47(36):16191621.

5. Warren WD, Zeppa R, Fomon JJ - Selective transsplenic decompression of gastroesophageal varices by distal splenorenal shunt. Ann Surg, 1967, 166(3): 437-455.

6. Lopes Filho GJ, Haddad CM - Late clinical, biochemical, endoscopic and electroencephalographic evaluation of patients with schistosomal portal hypertension treated with distal splenorenal shunt. Int Surg, 1998, 83(1):42-47.

7. Jenkins RL, Gedaly R, Pomposelli JJ, et al. - Distal splenorenal shunt: role, indications, and utility in the era of liver transplantation. Arch Surg, 1999, 134(4):416-420.

8- Antunes RA, Araújo ID - Evolução das varizes esofagogástricas após anastomose esplenorrenal proximal versus esplenorrenal distal. Rev Col Bras Cir, 2000, 27(2):102-108.
9. Abrantes WL, Vieira JN, Fernandes AD, et al. Embolização da artéria esplênica para tratamento de "cor pulmonale" agudo secundário à descompressão portal seletiva em paciente com forma hepatosplênica da esquistossomose e hipertensão pulmonar. AMB Rev Assoc Méd Bras, 1981, 27(3):110.

10. Zannini G, Masciariello S, Pagano G, et al. Percutaneous splenic artery occlusion for portal hypertension. A new mechanical technique for hypersplenism. Arch Surg, 1983, 118(8):897-900.

11. Ramires RP, Zils CK, Mattos AA - Escleroterapia versus somatostatina na hemorragia digestiva alta por ruptura de varizes esofágicas. Arq Gastroenterol, 2000, 37(3):148-154.

12. Maksoud JG, Gonçalves ME - Treatment of portal hypertension in children. World J Surg, 1994, 18(2):251258.

13. Raia S, da Silva LC, Gayotto LC, et al. - Portal hypertension in schistosomiasis: a long-term followup of a randomized trial comparing three types of surgery. Hepatology, 1994, 20(2):398-403.

14. Jin GL, Rikkers LF - The significance of portal vein thrombosis after distal splenorenal shunt. Arch Surg, 1991, 126(8):1011-1016.

Endereço para correspondência:

Valdinaldo Aragão de Melo

Rua Álvaro Brito, 14, apt 301

Praia 13 de Julho

CEP: 49020-400 - Aracaju - SE - Brasil

E-mail: vamelo@infonet.com.br 